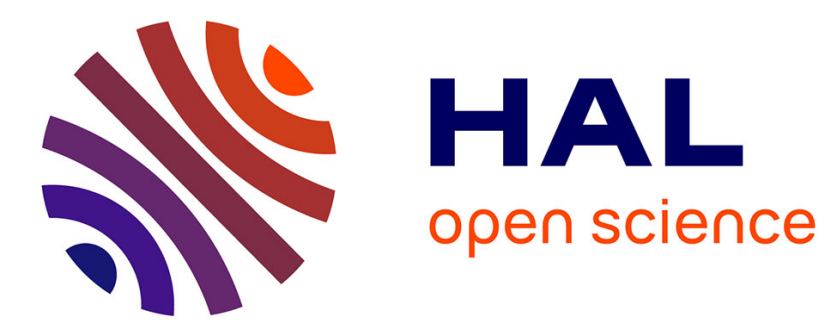

\title{
Characterization and Performance Analysis of a Chiral-Metamaterial Channel with Giant Optical Activity for Terahertz Communications
}

\author{
Anna Maria Vegni, Valeria Loscrì
}

\section{- To cite this version:}

Anna Maria Vegni, Valeria Loscrì. Characterization and Performance Analysis of a ChiralMetamaterial Channel with Giant Optical Activity for Terahertz Communications. Nano Communication Networks, 2016, 9, pp.28-35. 10.1016/j.nancom.2016.07.004 · hal-01379904

\section{HAL Id: hal-01379904 \\ https://inria.hal.science/hal-01379904}

Submitted on 14 Oct 2016

HAL is a multi-disciplinary open access archive for the deposit and dissemination of scientific research documents, whether they are published or not. The documents may come from teaching and research institutions in France or abroad, or from public or private research centers.
L'archive ouverte pluridisciplinaire HAL, est destinée au dépôt et à la diffusion de documents scientifiques de niveau recherche, publiés ou non, émanant des établissements d'enseignement et de recherche français ou étrangers, des laboratoires publics ou privés. 


\title{
Characterization and Performance Analysis of a Chiral-Metamaterial Channel with Giant Optical Activity for Terahertz Communications
}

\author{
Anna Maria Vegni ${ }^{\mathrm{a}, *}$, Valeria Loscrí ${ }^{\mathrm{b}}$ \\ ${ }^{a}$ Department of Engineering, Roma Tre University, Rome, Italy \\ ${ }^{b}$ INRIA Lille-Nord Europe, FUN Research Lab, Lille Nord-Europe, France.
}

\section{Abstract}

Technology in the $\mathrm{THz}$ frequency band has progressed rapidly in the last few years. The $\mathrm{THz}$ frequency band offers greater communication bandwidth than microwaves frequencies, and is becoming a standard for nanoscale communications. Traditional channel models for lower frequencies do not take into consideration specific properties as the very high molecular absorption or the very high reflection loss. In addition, in a propagation medium exhibiting a Giant Optical Activity, it is also important to derive the characteristics of the channel affected by chirality effects. This phenomenon occurs in particular material known as chiralmetamaterials in the (4-10) $\mathrm{THz}$ band.

The main contribution of this paper consist in the analysis of specific parameters of a chiral-metamaterial, such as the relative electrical permittivity, magnetic permeability and chirality coefficients. These parameters are considered for the channel model derivation both in Line-of-Sight and No Line-of-Sight propagation.

The chiral effect affects the channel through the presence of spectral windows, due to peaks of resonance of chiral parameter. Performance analysis of the chirality-affected channel is assessed in terms of $(i)$ channel capacity, (ii) propaga-

\footnotetext{
${ }^{*}$ Corresponding author

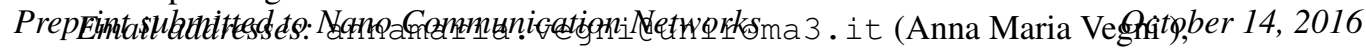
valeria.loscrieinria.fr (Valeria Loscrí)
} 
tion delay, (iii) coherence bandwidth, and (iv) symbol rates, for different distances and propagation modes.

Keywords: THz band, chirality effects, Giant Optical Activity, nano-communications

\section{Introduction}

Over the last few years we have witnessed an increasing demand for much higher speed and ubiquitous wireless communication systems. Technological development pave the advent of new communication paradigms, such as the Internet of NanoThings [1]. Following this trend, the THz frequency band is rising as a very promising solution to enable ultra-high-speed communications with the aim to overcome the spectrum scarcity and capacity limitations of current wireless systems. Advanced physical layer solutions are required, able to capture the specific and inherent features of the $\mathrm{THz}$ frequency bands. Indeed, traditional channel 0 models for lower frequency bands cannot be adopted for $\mathrm{THz}$ communication. 1 Some new channel models have been presented for THz frequency bands [2, $3,4,5,6]$, where some of the specific characteristics of this frequency band, such 3 as the high molecular absorption and the spreading loss, are taken into consider4 ation and analyzed. Also, the analysis of signal propagation has been addressed 5 in [7] through a multi-ray approach by assuming reflected, scattered and diffracted 6 paths. As a result, the authors derived the main THz-band channel features, such 7 as the distance-varying spectral windows, and the temporal broadening effects.

8 As it can be noticed from previous works, channel modeling in $\mathrm{THz}$ band 9 is typically addressed through the study of its transfer functions that consider 20 specific features, like molecular absorption loss or spreading loss, among oth- 
1 ers. However, the previous models take into consideration some specific features 2 of the channel at $\mathrm{THz}$ frequencies, but neglect other important effects that could 3 play a very important role in the channel modeling. One of these properties is 4 the electro-magnetic chirality effect and the specific features of the propagation 5 medium. Chirality effect is the characteristic of some natural materials to ren6 der an electric/magnetic response (displacement) under a magnetic/electric exci7 tation (field), respectively. This effect can be recognized in the so-called natu$8 \mathrm{ral} /$ artificial chiral materials. Examples of natural chiral materials are the sugar 9 molecules (sucrose) or the cholesteric liquid crystals. Artificial chiral materials, 10 e.g., sculptured thin films [8], can be obtained by doping a natural dielectric with 11 an amount of metallic/dielectric impurities each working as a couple of interacting 12 electro-magnetic dipoles. These impurities generate the handedness experimen13 tal evidence that prevents the superimposition of the molecular structure over a 14 reverse copy of itself.

15 The relative chirality parameter is an intrinsic characteristic of a chiral homo16 geneous isotropic medium. Standard values are in the range $[0,1]$. We observe 17 that in a specific range of the $\mathrm{THz}$ band, i.e., (4-10) $\mathrm{THz}$, the chiral parameter can 18 reach very high values that vary with the frequency, and show a resonant behav19 ior. Materials with this specific feature are said to exhibit a Giant Optical Activity 20 (GOA). In particular media such as the chiral complex materials where a GOA 21 takes place, the relative chirality parameter is complex and frequency-dependent, 22 showing multiple peaks at specific resonance frequencies [9].

23 GOA material affected by chirality effects is generally called as chiral-metamaterial [10], 24 due to the effect that the real part of relative electric permittivity and magnetic 25 permeability of the material shows negative values, and are frequency-dependent. 
1 This interesting property is of great interest to many areas of science, like analyt2 ical chemistry and molecular biology. Finally, chiral-metamaterials are suitable 3 media for the realization of nanosystem applications. Specifically, they represent 4 the ideal candidates for operations in the THz band. In fact, in comparison to nat5 ural materials, chiral-metamaterials show a strong response to the $\mathrm{THz}$ radiation 6 that represents a great technological potential in several sectors such as imaging, 7 sensing, and also communications.

8 In this paper, we focus on GOA chiral-metamaterials, derive the channel trans9 fer function and analyze its specific behavior in case of direct and multi-path prop10 agation, in the (4-10) $\mathrm{THz}$ frequency range [9]. Due to the resonant behavior of 11 chiral parameter, the channel model shows specific frequency-dependent spectral 12 windows, guaranteeing high bandwidth values.

13 This paper is organized as follows. In Section 2 we introduce the frequency14 dependent behavior of specific parameters of a chiral-metamaterial. Starting from 15 the concept of electro-magnetic chirality $[11,12,13]$, we consider the chiral ef16 fects following the change in the propagation velocity and in the refractive index, 17 due to the chiral impurities inside the propagation medium. These effects are eval18 uated also in the case when the considered medium exhibits a GOA $[9,14]$. Then, 19 in Section 3 we derive the corresponding chirality-affected channel model for $\mathrm{THz}$ 20 band, and present the related frequency-dependent spectral windows. Section 4 is 21 then devoted to the performances of the chiral channel, assessed in terms of ca22 pacity, propagation delay, coherence bandwidth, and symbol rates, in case of LoS 23 and NLoS propagation modes. Finally, conclusions are drawn at the end of the 24 paper. 


\section{Full-Wave Propagation Model in a Chiral-Metamaterial}

2 Starting from the classic harmonic macroscopic Maxwell's equations, we con3 sider the electro-magnetic propagation inside a generic complex material, under 4 the assumption that it is a linear and chiral medium.

5 Unconventional materials (i.e., metamaterials) are specifically considered since 6 the GOA is reinforced by using thin metallic crossed-structure impurities in the 7 host dielectric medium, that is chiral-metamaterials [10]. Therefore, we consider 8 a time-harmonic generic linear material, where chiral (magneto/electric-optical)

9 effects are included in the following constitutive relations, written as:

$$
\left\{\begin{array}{l}
\mathbf{B}=\underline{\xi} \bullet \mathbf{E}+\underline{\mu} \bullet \mathbf{H} \\
\mathbf{D}=\underline{\varepsilon} \bullet \mathbf{E}+\underline{\zeta} \bullet \mathbf{H}
\end{array}\right.
$$

11 where we remind that $\mathbf{B}$ is the magnetic displacement and $\mathbf{H}$ is the magnetic field, 12 as well as $\mathbf{D}$ is the electric displacement and $\mathbf{E}$ is the electric field. Finally, the 13 symbol $\bullet$ represents the scalar product operator, and $\underline{\xi}, \underline{\mu}, \underline{\varepsilon}$, and $\underline{\zeta}$ are specific 14 tensor quantities of the material.

15 From (1) we observe the chirality property through the dependence of $(i) \mathbf{E}$ 16 in $\mathbf{B}$, and (ii) $\mathbf{H}$ in $\mathbf{D}$. Furthermore, the displacement field existing inside the 17 material is generated by an excitation expressed in terms of intensity of the inci18 dent electro-magnetic field. Therefore, the material under consideration is a linear 19 chiral medium.

20 We remind that the chiral effects have a two-fold meaning, i.e., $(i)$ an electric 21 field applied on the material provides not only an electric induction, but also a 22 magnetic displacement, and (ii) a magnetic field applied on the material provides 23 not only a magnetic induction, but also an electric displacement, unlike from non- 
1 chiral materials.

2 As previously said, in this paper our attention is devoted to GOA chiral3 metamaterials [15], where the authors show that for a GOA reciprocal material 4 the specific constitutive relations are as follows:

$$
\left[\begin{array}{l}
\mathbf{B} \\
\mathbf{D}
\end{array}\right]=\left[\begin{array}{cc}
-j \frac{\xi_{0} \xi_{r}}{c} & \mu_{0} \mu_{r} \\
\varepsilon_{0} \varepsilon_{r} & j \frac{\xi_{0} \xi_{r}}{c}
\end{array}\right] \bullet\left[\begin{array}{c}
\mathbf{E} \\
\mathbf{H}
\end{array}\right],
$$

6 where it is clear that the natural dielectric (where $\varepsilon_{b}$ and $\mu_{b}$ are the permittivity and 7 permeability, respectively) becomes a metamaterial. Moreover, according to [15], 8 at the frequency around $5 \mathrm{THz}$ and $8 \mathrm{THz}$, there are four resonance frequencies of 9 the $\xi_{r}$ relative chirality parameter, i.e., $[4.8,5.6,7.9,8.2] \mathrm{THz}$.

10 The same consideration is applied to the relative permittivity and permeability 11 parameters, still in the (4-10) $\mathrm{THz}$ band. As reported in $[9,16], \varepsilon_{r}$ and $\mu_{r}$ are 12 complex parameters, and the real part has a frequency-dependent behavior with 13 resonant peaks.

$$
\begin{aligned}
& {\left[\begin{array}{l}
\mathbf{B} \\
\mathbf{D}
\end{array}\right]=} \\
& =\left[\begin{array}{cc}
-j \frac{\Omega_{\xi} \omega_{0} \omega}{\omega_{0}^{2}-\omega^{2}-j \omega \gamma} & \mu_{0}\left(\mu_{b}+\frac{\Omega_{\mu} \omega^{2}}{\omega_{0}^{2}-\omega^{2}-j \omega \gamma}\right) \\
\varepsilon_{0}\left(\varepsilon_{b}+\frac{\Omega_{\varepsilon} \omega_{0}^{2}}{\omega_{0}^{2}-\omega^{2}-j \omega \gamma}\right) & j \frac{\Omega_{\xi} \omega_{0} \omega}{\omega_{0}^{2}-\omega^{2}-j \omega \gamma}
\end{array}\right] \bullet\left[\begin{array}{c}
\mathbf{E} \\
\mathbf{H}
\end{array}\right],
\end{aligned}
$$

16 where $\omega=2 \pi f, \varepsilon_{0}$, and $\mu_{0}$ are the absolute permittivity and permeability, respec- 
1 tively. Then, the constitutive parameters of interest are:

2

$$
\varepsilon=\varepsilon_{0} \varepsilon_{r}=\varepsilon_{0}\left[\varepsilon_{b}+\Omega_{\varepsilon} \omega_{0}^{2}\left(\frac{\omega_{0}^{2}-\omega^{2}+j \omega \gamma}{\left(\omega_{0}^{2}-\omega^{2}\right)^{2}+\omega^{2} \gamma^{2}}\right)\right]
$$

$$
\mu=\mu_{0} \mu_{r}=\mu_{0}\left[\mu_{b}+\Omega_{\mu} \omega^{2}\left(\frac{\omega_{0}^{2}-\omega^{2}+j \omega \gamma}{\left(\omega_{0}^{2}-\omega^{2}\right)^{2}+\omega^{2} \gamma^{2}}\right)\right],
$$

$$
\xi_{r}=\Omega_{\xi} \omega_{0} \omega\left(\frac{\omega_{0}^{2}-\omega^{2}+j \omega \gamma}{\left(\omega_{0}^{2}-\omega^{2}\right)^{2}+\omega^{2} \gamma^{2}}\right) .
$$

7 It is noted that the frequency resonant behavior of $\xi_{r}$ is a Drude-like one, and $\Omega_{\varepsilon}$, $8 \Omega_{\mu}, \Omega_{\xi}, \gamma$, and $\omega_{0}$ are specific parameters of the GOA material [15].

By simple computations, and assuming a lossy material, i.e.,

11 we obtain

$$
\left\{\begin{aligned}
\varepsilon_{b} & =\operatorname{Re}\left[\varepsilon_{b}\right]+j \operatorname{Im}\left[\varepsilon_{b}\right] \\
\mu_{b} & =\operatorname{Re}\left[\mu_{b}\right]+j \operatorname{Im}\left[\mu_{b}\right]
\end{aligned}\right.
$$

$$
\xi_{r}=\omega_{0} \Omega_{\xi}\left[\frac{\omega\left(\omega_{0}^{2}-\omega^{2}\right)}{\left(\omega_{0}^{2}-\omega^{2}\right)^{2}+\omega^{2} \gamma^{2}}\right]+j \omega_{0} \Omega_{\xi}\left[\frac{\gamma \omega^{2}}{\left(\omega_{0}^{2}-\omega^{2}\right)^{2}+\omega^{2} \gamma^{2}}\right] .
$$


1 dex:

2

$$
\begin{aligned}
& \varepsilon_{r} \mu_{r}+\xi_{r}^{2}=\left(\operatorname{Re}\left[\varepsilon_{r}\right]+j \operatorname{Im}\left[\varepsilon_{r}\right]\right)\left(\operatorname{Re}\left[\mu_{r}\right]+j \operatorname{Im}\left[\mu_{r}\right]\right)+ \\
& +\left(\operatorname{Re}\left[\xi_{r}\right]+j \operatorname{Im}\left[\xi_{r}\right]\right)^{2}>0,
\end{aligned}
$$

we observe that the following conditions hold for a GOA material:

$$
\Omega_{\varepsilon}=\left(1+\frac{\gamma^{2}}{\omega^{2}-\omega_{0}^{2}}\right) \operatorname{Re}\left[\varepsilon_{b}\right]
$$

$$
\begin{gathered}
\Omega_{\mu}=\left(1+\frac{\gamma^{2}}{\omega^{2}-\omega_{0}^{2}}\right) \operatorname{Re}\left[\mu_{b}\right], \\
\Omega_{\xi}=\left(1+\frac{\gamma^{2}}{\omega^{2}-\omega_{0}^{2}}\right) \sqrt{\operatorname{Re}\left[\varepsilon_{b}\right] \operatorname{Re}\left[\mu_{b}\right]} .
\end{gathered}
$$

7 It is observed that relations (12), (13), and (14) state the connection between the 8 host material parameters, i.e., $\varepsilon_{b}$, and $\mu_{b}$, and the specific GOA ones in order to 9 obtain a positive refractive index in (11).

10 Finally, the computation of the electro-magnetic field in the channel is carried 11 out through the solutions of linear differential equations, arising from the follow12 ing source-less Maxwell's equations:

$$
\left\{\begin{array}{l}
(\underline{\nabla}+j \omega \underline{\xi}) \bullet \mathbf{E}=-j \omega \underline{\mu} \bullet \mathbf{H} \\
(\underline{\nabla}-j \omega \underline{\zeta}) \bullet \mathbf{H}=j \omega \underline{\varepsilon} \bullet \mathbf{E}
\end{array}\right.
$$

14 and the solving differential equations for $\mathbf{E}$ and $\mathbf{H}$ are given, respectively:

15

$$
\left\{\begin{array}{l}
{\left[(\underline{\nabla}-j \omega \underline{\zeta}) \bullet \underline{\mu}^{-1} \bullet(\underline{\nabla}+j \omega \underline{\xi})-\omega^{2} \underline{\varepsilon}\right] \bullet \mathbf{E}=0} \\
{\left[(\underline{\nabla}+j \omega \underline{\xi}) \bullet \underline{\varepsilon}^{-1} \bullet(\underline{\nabla}-j \omega \underline{\zeta})-\omega^{2} \underline{\mu}\right] \bullet \mathbf{H}=0}
\end{array}\right.
$$

16 where $\underline{\nabla}$ is Kong's operator. 
As a noteworthy point, we can examine how the constitutive relations influ2 ence the channel polarization properties. Typically, the antenna at the transmitter 3 side is a radiating element that can be linearly or circularly polarized. In these 4 two cases we obtain interesting features of the channel directly connected with 5 the constitutive relations of the medium. Namely, by assuming the linear polar6 ization of the electric field generated by an appropriate antenna (i.e., $\mathbf{E}=E_{x} \hat{\mathbf{x}}$ ), 7 we obtain the final second order partial differential equation for $E_{x}$, i.e.,

$$
\alpha_{1} E_{x}+\alpha_{2} \frac{\partial E_{x}}{\partial x}+\alpha_{3} \frac{\partial E_{x}}{\partial y}+\alpha_{4} \frac{\partial E_{x}}{\partial z}+\alpha_{5} \frac{\partial^{2} E_{x}}{\partial y^{2}}+\alpha_{6} \frac{\partial^{2} E_{x}}{\partial z^{2}}=0
$$

9 where $\alpha_{i}$ with $i=(1,2, \ldots, 6)$ are coefficients depending on the elements of $\underline{\varepsilon}, \underline{\mu}$, $10 \underline{\xi}$, and $\underline{\zeta}$ tensors.

11 On the other side, for a circularly polarized antenna, i.e., $\mathbf{E}=E_{x} \hat{\mathbf{x}}+E_{y} \hat{\mathbf{y}}=$ $12 E_{x} \hat{\mathbf{x}}+j( \pm) E_{x} \hat{\mathbf{y}}$, the final second order partial differential equation for $E_{x}$ is 13 obtained:

$14 \quad \gamma_{1} E_{x}+\gamma_{2} \frac{\partial^{2} E_{x}}{\partial x^{2}}+\gamma_{3} \frac{\partial^{2} E_{x}}{\partial x \partial y}+\gamma_{4} \frac{\partial^{2} E_{x}}{\partial x \partial z}+\gamma_{5} \frac{\partial^{2} E_{x}}{\partial y^{2}}+\gamma_{6} \frac{\partial^{2} E_{x}}{\partial y \partial z}+\gamma_{7} \frac{\partial^{2} E_{x}}{\partial z^{2}}=0$ where $\gamma_{j}$ with $j=(1,2, \ldots, 7)$ are coefficients depending on the elements of $\underline{\varepsilon}$, $\underline{\mu}, \underline{\xi}$, and $\underline{\zeta}$ tensors. Equations (17) and (18) allow to classify the transmission 17 properties of the channel according to the choice of the transmitting medium. 18 Therefore, such formulas are useful as design tools for materials working in $\mathrm{THz}$ band, including GOA materials and optical metamaterials.

$20 \quad$ Notice that $\alpha_{i}$ and $\gamma_{j}$ parameters can be described through 36 degrees of free$21 \mathrm{dom}$ as a consequence of their dependance on $\underline{\varepsilon}, \underline{\mu}, \underline{\xi}$, and $\underline{\zeta}$ tensors. This means 22 that we have a lot of partial differential linear equations similar to (17) and (18) 
1 that should be examined in order to determine the corresponding propagation char-

2 acteristics of the channel. However, as future work, we can investigate this point

3 by starting from a topology of channel material (e.g., bianisotropic, biaxial, etc.)

4 and then determine the specific transmission/ reflection related properties.

5

\section{Chiral-affected Channel Model}

In this section, we present how the relative chiralilty parameter affects the channel performance in the (4-10) $\mathrm{THz}$ band, in the case of ray tracing propagation (i.e., LoS, and NLoS), and under the linear polarization hypothesis. Specifically, in NLoS case, we focus on reflected paths due at generic reflection centers located at $z$-plane. The reflection characteristics of the transmissive channel can be evaluated through the specific knowledge of the local planar geometry associated to the reflection centers.

The use of ray tracing techniques for channel modeling in $\mathrm{THz}$ band has been largely adopted, like in [5], where Han et al. consider a multi-ray approach with one direct path, and other reflected, scattered, and diffracted paths. According to this approach, the channel model is the combination of several individual narrow sub-bands, each of them with a flat-band response. Assuming $N_{i}$ narrow subbands, and in the case of stationary environment, the channel response in the $i$-th sub-band is given as

$$
h_{i}(\tau)=\sum_{n=1}^{N_{i}} \alpha_{i, n} \delta\left(\tau-\tau_{n}\right),
$$

where $\alpha_{i, n}$ is the frequency-dependent attenuation, and $\tau_{n}$ is the propagation delay of the $n$-th ray in the multi-ray approach.

From (19), and according to the computations in [5], we can derive the LoS 
1 and NLoS channel transfer functions in the case of chirality-affected channel with 2 GOA, respectively as:

$$
H_{\mathrm{LoS}}(f)=H_{\mathrm{Abs}}(f) H_{\mathrm{Spr}}(f) e^{-j 2 \pi f \tau_{\mathrm{LoS}}},
$$

4 and

$$
H_{\mathrm{NLoS}}(f)=\left[\frac{\nu_{c}}{4 \pi f\left(d_{1}+d_{2}\right)}\right] e^{-j 2 \pi f \tau_{\mathrm{NLoS}}-\frac{1}{2} k(f)\left(d_{1}+d_{2}\right)} \cdot R(f),
$$

6 where we assume the NLoS scenario is affected by reflected rays only, through 7 the rough surface reflection loss, i.e., $R(f)$.

In (20), $H_{\mathrm{Abs}}$ is the transfer function due to the molecular absorption loss, while $H_{\text {Spr }}$ is the spreading loss that takes account for the chirality effect through $10 \nu_{c}$ that is the propagation velocity of the electro-magnetic field in a chiral homo11 geneous isotropic medium, i.e.,

$$
\nu_{c}=\frac{c}{n_{c}}
$$

13 where $c$ is the light propagation speed, and $n_{c}$ is the refractive index in a chiral 14 medium, i.e.,

$$
n_{c}=\sqrt{\mu_{r} \varepsilon_{r}+\xi_{r}^{2}}
$$

16 with $\mu_{r}, \varepsilon_{r}$ and $\xi_{r}$ frequency-dependent parameters, as depicted in [16]. Finally, 17 under the hypothesis of stationary scenario where the transmitter and the receiver 18 are at a distance $d[\mathrm{~m}]$, from (20) we obtain the propagation delay for the LoS ray 19 as:

$$
\tau_{\mathrm{LoS}}=\frac{d}{\nu_{c}}
$$

For the NLoS channel transfer function expressed in (21), by assuming $d_{1}$ as 


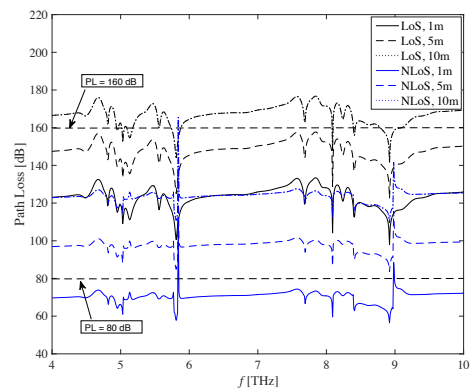

(a)

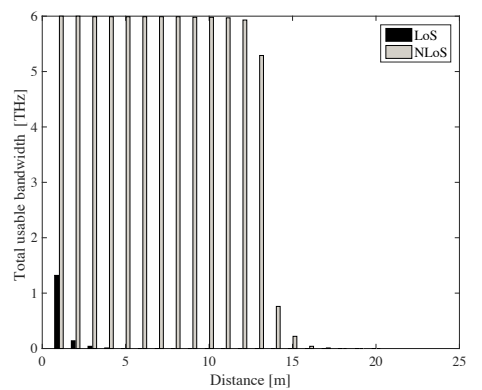

(b)

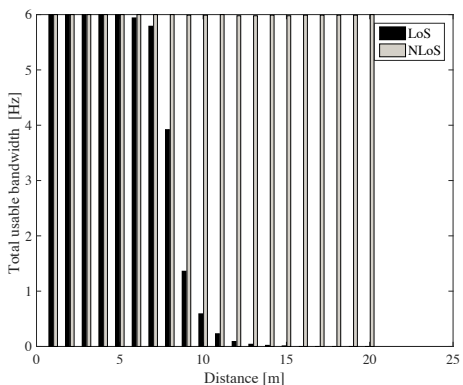

(c)

Figure 1: Spectral windows in a chiral-affected channel with GOA. (a) Path loss, and (b) total usage bandwidth for LoS and NLoS propagation in case of a path loss threshold of $120 \mathrm{~dB}$, and (c) $160 \mathrm{~dB}$.

1 the distance between the transmitter and a generic reflecting point, and $d_{2}$ as the

2 distance between this point and the receiver, we obtain the propagation delay of

3 the $j$-th NLoS ray along the distance $\left(d_{1}+d_{2}\right)$, as

$$
\tau_{\mathrm{NLOS}}=\frac{d_{1}+d_{2}}{\nu_{c}}
$$

From the expressions of channel transfer functions in (20) and (21), it is easy

6 to compute the total path loss, as depicted in Figure 1 (a) in case of LoS and NLoS

7 propagations, for different distances from transmitter to receiver, and assuming a

8 specific reflecting angle for multi-path. The expressions of path loss in LoS and

$9 \quad$ NLoS are respectively:

$$
A_{\mathrm{LoS}}=A_{s}+A_{a}=20 \log _{10}\left(\frac{4 \pi d}{\lambda_{\mathrm{Chir}}}\right)+10 \gamma d \log _{10} e
$$


1 and

$$
\begin{aligned}
& A_{\mathrm{NLOS}}=10 \log _{10}\left(\frac{\nu_{c}}{4 \pi f\left(d_{1}+d_{2}\right)}\right)+ \\
& +10 \log _{10}\left(e^{-\frac{1}{2} \alpha\left(d_{1}+d_{2}\right)} e^{-\frac{2 \cos \left(\beta_{i}\right)}{\sqrt{n_{c}^{2}-1}}} e^{-\frac{8 \pi^{2} f^{2} \sigma^{2} \cos ^{2}\left(\beta_{i}\right)}{\nu_{c}^{2}}}\right) .
\end{aligned}
$$

3 where $\lambda_{c}=\nu_{c} / f$ is the wavelength in the considered medium affected by the 4 homogeneous chirality, and $\gamma$ is the absorption coefficient measuring the amount 5 of absorption loss of the EM field in the medium.

6 From Figure 1, by increasing the distance, the path loss has a higher trend.

7 Moreover, similarly to the results in [7], the LoS propagation (black lines) pro8 vides higher values with respect to the NLoS scenario (blue lines). In both LoS 9 and NLoS, the behavior is frequency-dependent, but in LoS propagation the path 10 loss has a smoother trend, with some peaks at 5.8, 8.09 and $8.93 \mathrm{THz}$. On the other 11 hand, for NLoS propagation, the peaks are well noticeable at 5.8 and $8.93 \mathrm{THz}$, 12 while on the other frequencies, the trend is on average flat around 70, 100 and $13120 \mathrm{~dB}$ for $\operatorname{LoS}$ at $d=1,5$, and $10 \mathrm{~m}$, respectively.

14 Similarly to the analysis conducted in [7], we aim to characterize the spectral 15 windows of chiral channel transfer functions in case of LoS and NLoS propaga16 tion. A spectral window is given by the portion of spectrum below a given path 17 loss threshold. We expect to observe that the path loss peaks caused by the chiral 18 effect create several spectral windows, with different bandwidths in each of them. 19 In the case of a path loss threshold set to $80 \mathrm{~dB}$, the communication distance is 20 limited for NLoS propagation at lower distance of $1 \mathrm{~m}$, except the two peaks that 21 are above this threshold, and correspond to $5.84 \mathrm{THz}$ and $8.98 \mathrm{THz}$. According 22 to the values assumed in [7], the threshold of $80 \mathrm{~dB}$ corresponds to no gains of 23 transmission and reception antennas, and so to a multi-path propagation model. 
In order to identify the spectral windows for LoS propagation, we have to in2 crease the path loss threshold around $120 \mathrm{~dB}$, so that a few windows appear for 3 a distance of $1 \mathrm{~m}$ (see curve $\operatorname{LoS}$ for $d=1 \mathrm{~m}$ in Figure $1(a)$ ). However, in 4 this case most of the path loss for LoS propagation is above the threshold, thus 5 providing a reduced usable bandwidth. Figure $1(b)$ depicts the usable bandwidth 6 versus the distance for the path loss threshold of $120 \mathrm{~dB}$. We notice that the avail7 able spectrum in LoS propagation is limited up to $4 \mathrm{~m}$, reaching a maximum at $81.32 \mathrm{THz}$ corresponding to a distance of $1 \mathrm{~m}$. In contrast, the NLoS propagation 9 reaches higher bandwidths, and then it decreases at $17 \mathrm{~m}$ where the bandwidth is $10 \quad 0.01 \mathrm{THz}$. The bandwidth rate in LoS is $75.5 \mathrm{GHz} / \mathrm{m}$, while it reaches $3.90 \mathrm{THz} / \mathrm{m}$ 11 in NLoS scenario.

12 It follows that an increase of the path loss threshold to $160 \mathrm{~dB}$ is expected 13 to provide higher values of usable bandwidth in LoS propagation, as depicted in 14 Figure $1(c)$. Higher path loss thresholds rise from higher antenna gains, and the 15 transmission becomes directional through the LoS path. In this case, the usable 16 bandwidth in LoS propagation reaches higher values than those for the threshold 17 of $120 \mathrm{~dB}$. The lowest value is $0.01 \mathrm{THz}$ for a distance of $15 \mathrm{~m}$, and the average 18 bandwidth rate for LoS propagation is $2.39 \mathrm{THz} / \mathrm{m}$.

19 On the other side, for NLoS propagation, the usable bandwidth reaches ap20 proximately the maximum value of $6 \mathrm{THz}$ for different distances, and then the 21 average rate of the total usable bandwidth is $5.99 \mathrm{THz} / \mathrm{m}$. As a result, we can 22 conclude that within the range (4-10) $\mathrm{THz}$ the available bandwidth is almost the 23 entire band, especially for NLoS propagation.

24 Notice that in this paper, we focus only on the transfer functions in LoS and 25 NLoS scenarios in a chirality-affected channel, assuming a flat behavior for the 
1 molecular absorption loss (frequency independent behavior). Then, in all the 2 simulation results we omit the frequency-dependent molecular absorption effect. 3 Specifically, in NLoS we assume the presence of reflected rays only, since we are 4 interested in the behavior of highly frequency-dependent reflections that depend 5 on the shape, material, and roughness of the reflecting surface affects the $\mathrm{THz}$ $6 \quad$ wave propagation.

\section{4. Chiral Channel Characterization}

$8 \quad$ Following the chirality-affected channel model presented in Section 3, in this 9 section we investigate its main features in the (4-10) THz band. Specifically, we 10 aim to characterize ( $i$ ) the channel capacity, (ii) the propagation delay, (iii) the 11 coherence bandwidth, and (iv) the symbol rate.

\section{4.1. Channel capacity and propagation delay}

13 To evaluate the capacity limits in a chiral medium, we refer to the approach 14 adopted in [5], where the received signal has been decomposed as a sum of the 15 sub-bands, each one with a narrow behavior and a flat-band response. The follow16 ing constraint is adopted:

$$
\sum_{i=1}^{N_{B}} P_{i} \leq P_{T O T},
$$

18 where $N_{B}$ is the total number of sub-bands, $P_{i}$ is the transmission power in the $i$ 19 th sub-band, and $P_{T O T}$ is the total transmit power in the (4-10) THz band. Notice 20 that, since the chiral parameter has a frequency-dependent behavior in (4-10) $\mathrm{THz}$ 21 band, we consider only this frequency range.

1 For $N_{B}$ sub-bands, the capacity can be defined as the sum of the single capac- 


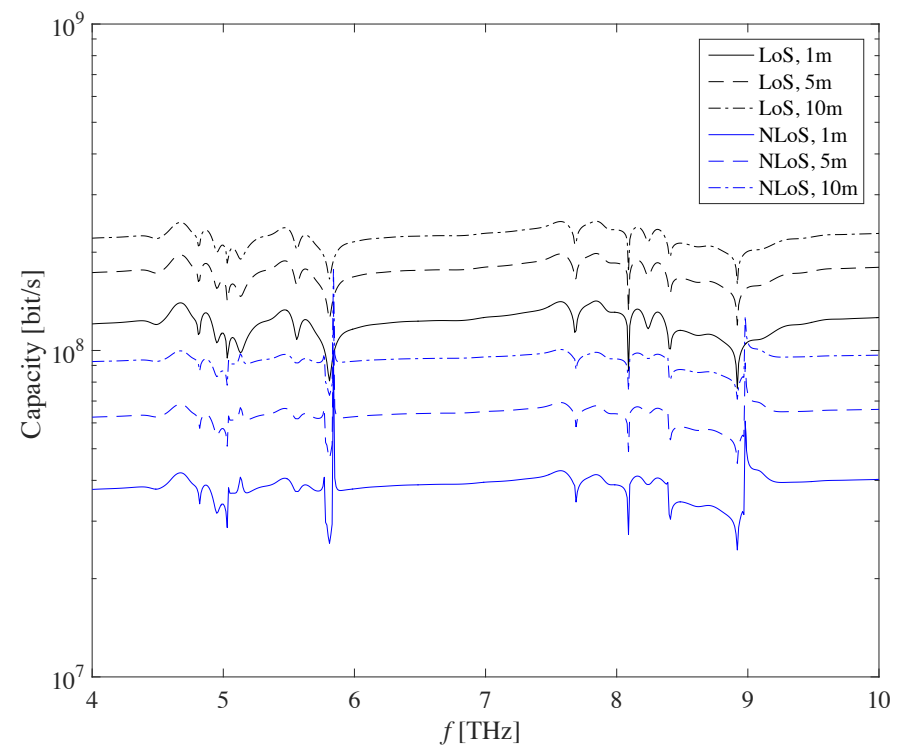

Figure 2: Capacity per sub-band of a chirality-affected channel with GOA, versus frequency for LoS and NLoS propagation, and different distances.

2 ities in each sub-band, i.e.,

$$
C=\sum_{i=1}^{N_{B}} C_{i}=\sum_{i=1}^{N_{B}} \Delta f_{i} \log \left(1+\frac{\left|h_{i}\right|^{2} P_{i}}{\Delta f_{i} S_{N}\left(f_{i}\right)}\right)
$$

4 where $S_{N}$ is the power spectral density of the additive white Gaussian noise, and $5 \Delta f_{i}$ is the sub-band range among two consecutive sub-bands, i.e., $\Delta f_{i}=f_{i+1}-f_{i}$, 6 assumed as $10 \mathrm{GHz}$ in our simulation results. We assume a flat power profile, that 7 is the total power transmission is uniformly distributed over the entire operative 8 band (i.e., from 4 to $10 \mathrm{THz}$ ). Also, we consider a power level of $46 \mathrm{dBm}$, divided $9 \quad$ across all the sub-bands, i.e., $N_{B}=600$.

Figure 2 depicts the chirality-affected channel capacity with GOA in case of 11 LoS and NLoS scenario. We notice that with a reduction of distance, the capacity 1 decreases as well, and also the LoS scenario has a smoother behavior with respect 


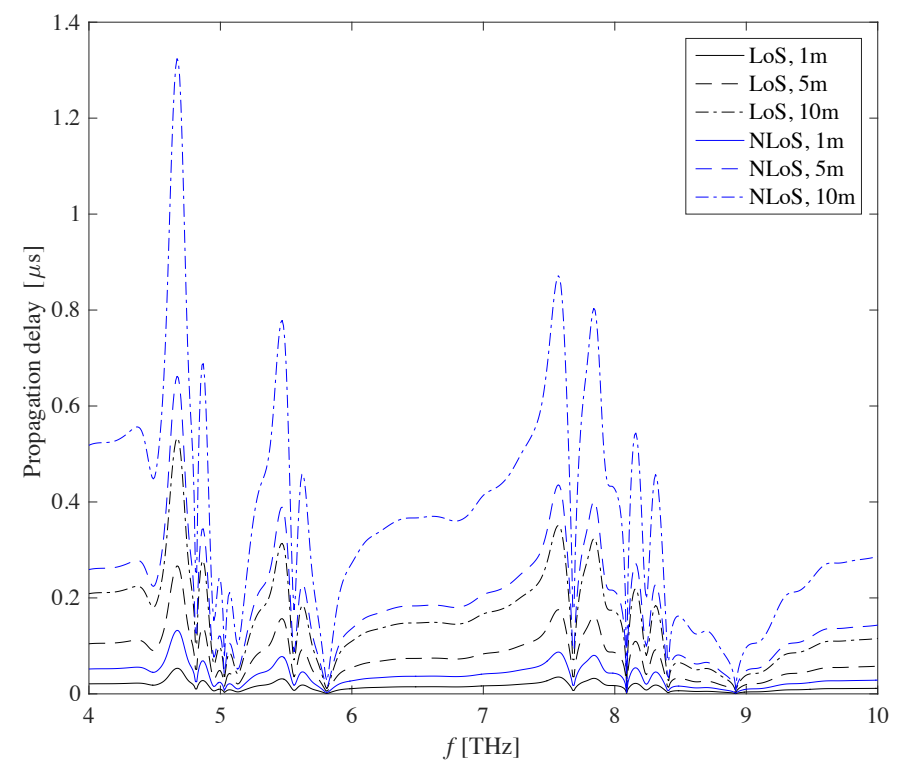

Figure 3: Propagation delay versus frequency in a chirality-affected channel with GOA, for LoS and NLoS propagation, and different distances.

2 to the frequency, while the NLoS shows an accentuate frequency-dependent trend, 3 with distinguishable peaks at resonance frequencies. Specifically, in LoS, the 4 capacity has an almost flat behavior, with a mean value of $0.22 \mathrm{Gbit} / \mathrm{s}$ for $d=$ $510 \mathrm{~m}$. Performances get worst in the case of NLoS propagation for $d=1 \mathrm{~m}$, 6 where we observe a degradation of capacity at 5.81 and $8.92 \mathrm{THz}$, corresponding 7 to $25.65 \mathrm{Mbit} / \mathrm{s}$ and $24.47 \mathrm{Mbit} / \mathrm{s}$, respectively. scenarios is depicted in Figure 3. We observe the frequency-dependent behavior 10 due to the chiral effect, and as expected, performance gets worst when the distance 11 increases. An almost-flat behavior is shown for LoS at short distances (i.e., $d=$ $121 \mathrm{~m}$ ), while a resonant trend appears when increasing the distance, as well as in 13 NLoS scenario due to the longer distances covered. Finally, we observe that the 1 propagation delay both in LoS and NLoS case shows lower values corresponding 


\subsection{Coherence bandwidth and symbol rate}

4

5

6

$7 \quad$ where $\overline{\tau_{i}}$ and $\bar{\tau}_{i}^{2}$ are the first and second moments of the instantaneous power-delay

The root mean square (rms) delay spread is a measure of how dispersive the channel is. It is expressed as [7]:

$$
\sigma_{i}=\sqrt{\overline{\tau_{i}^{2}}-\bar{\tau}_{i}^{2}}
$$

8 profile, respectively. From (30) we can derive information about the coherence

bandwidth, defined as the range of frequencies over which the channel correlation exceeds $50 \%$.

In our simulations, we consider two scenarios with a variable number of NLoS reflected rays, i.e., (i) one, and (ii) five, and one direct ray. In both cases, we observe the frequency-dependent behavior as typical of chiral materials exhibiting GOA. In Figure $4(a)$ we show the coherence bandwidth in the case of one LoS and one NLoS path for different distances. As experienced in [5], higher values are reached for shorter distances. However, we cannot compare our results to others obtained with pre-existing approaches, since the frequency range is not the same.

In our simulations, several peaks appear due to the chirality effects. This can allow tuning the frequency to resonant peaks in order to obtain higher performances. For example, for $d=1 \mathrm{~m}$, the minimum value of rms delay is $0.33 \mathrm{ns,}$ corresponding to $8.92 \mathrm{THz}$. This value corresponds to a symbol rate limited to $0.1 / \sigma_{i}=0.29 \mathrm{Gbit} / \mathrm{s}$ to avoid inter-symbol interference. Also, in this case, the coherence bandwidth is limited to $0.59 \mathrm{GHz}$ at the frequency peak of $8.92 \mathrm{THz}$. 
2 On the other side, when the distance increases (i.e., $d=10 \mathrm{~m}$ ), the minimum 3 rms delay is $3.6 \mathrm{~ns}$ at $8.92 \mathrm{THz}$. This value provides a symbol rate limited to $427.71 \mathrm{Mbit} / \mathrm{s}$, and the coherence bandwidth equals to $55.43 \mathrm{MHz}$, still at the same 5 frequency.

Performances get worst in case of multiple reflected paths, as shown in Fig-

7 ure $4(b)$. For $d=1 \mathrm{~m}$ the minumum $\mathrm{rms}$ is $0.78 \mathrm{~ns}$, which corresponds to a 8 coherence bandwidth of $0.25 \mathrm{GHz}$. For higher distances (i.e., $d=10 \mathrm{~m}$ ) the 9 minimum rms is $7.31 \mathrm{~ns}$, corresponding to a coherence bandwidth is $27.3 \mathrm{MHz}$.

10 Finally, following the analysis of coherence bandwidths, we can derive the 11 symbol rates in different scenarios, as depicted in Figure $5(a)$ and $(b)$ in case of 12 (a) one LoS and one NLoS path, and $(b)$ one LoS and five reflected NLoS paths, 13 respectively. We notice the symbol rate is limited to a maximum of $0.29 \mathrm{Gbit} / \mathrm{s}$ 14 corresponding to $8.92 \mathrm{THz}$, in order to avoid InterSymbol Interference (ISI) for 15 linearly-modulated signals. Again, the chiral frequency behavior is observed, and 16 a decrease of symbol rate is experienced for increasing distances (e.g., for $d=$ $1710 \mathrm{~m}$ the symbol rate reaches $0.27 \mathrm{Mbit} / \mathrm{s}$ at $4.67 \mathrm{THz}$ ). Finally, as depicted in 18 Figure $5(b)$ an increase of reflected NLoS paths affects the symbol rate, then 19 causing a decrease of performances until $0.13 \mathrm{Mbit} / \mathrm{s}$ for $d=10 \mathrm{~m}$ at $4.67 \mathrm{THz}$, 20 while the maximum value is $0.12 \mathrm{Gbit} / \mathrm{s}$ obtained for $d=1 \mathrm{~m}$ at the frequency of $21 \quad 8.92 \mathrm{THz}$.

\section{Conclusions}

In this paper we have derived the channel transfer function of a GOA chirality 24 affected channel, both in the case of LoS and NLoS propagation in the (4-10) THz 1 band. 


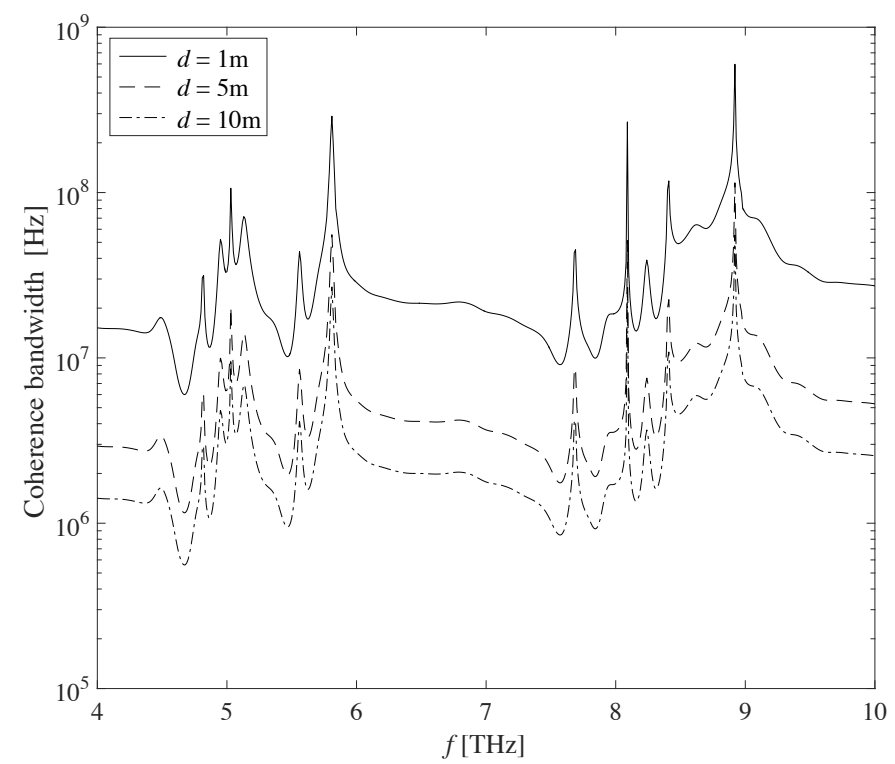

(a)

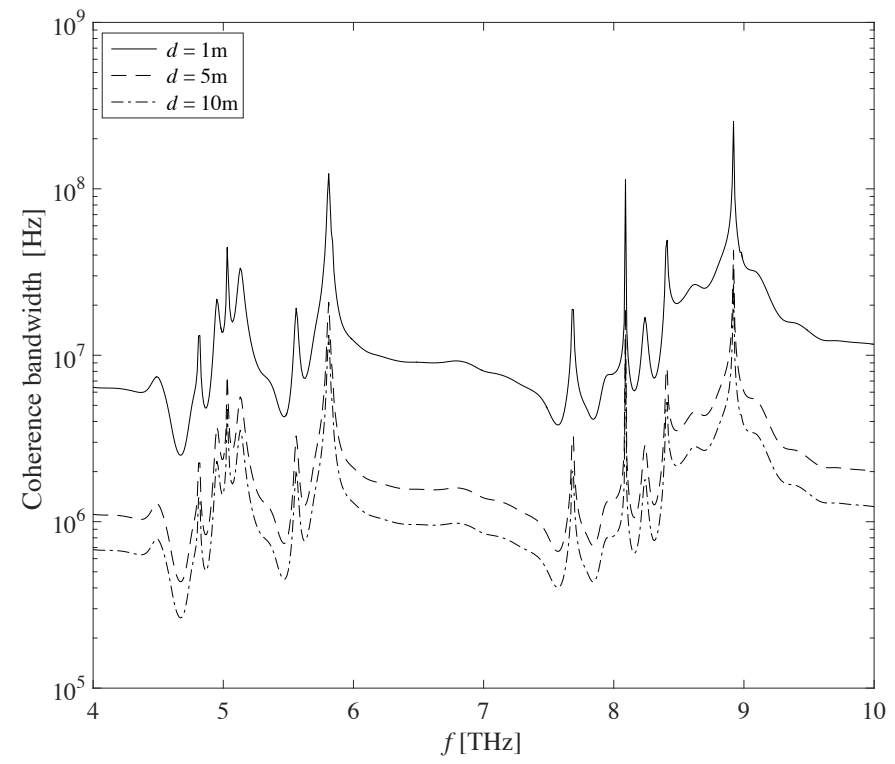

$(b)$

Figure 4: Coherence bandwidth in a chirality-affected channel with GOA, in case of (a) one LoS and one NLoS path, an $(b)$ one LoS and five NLoS paths. 


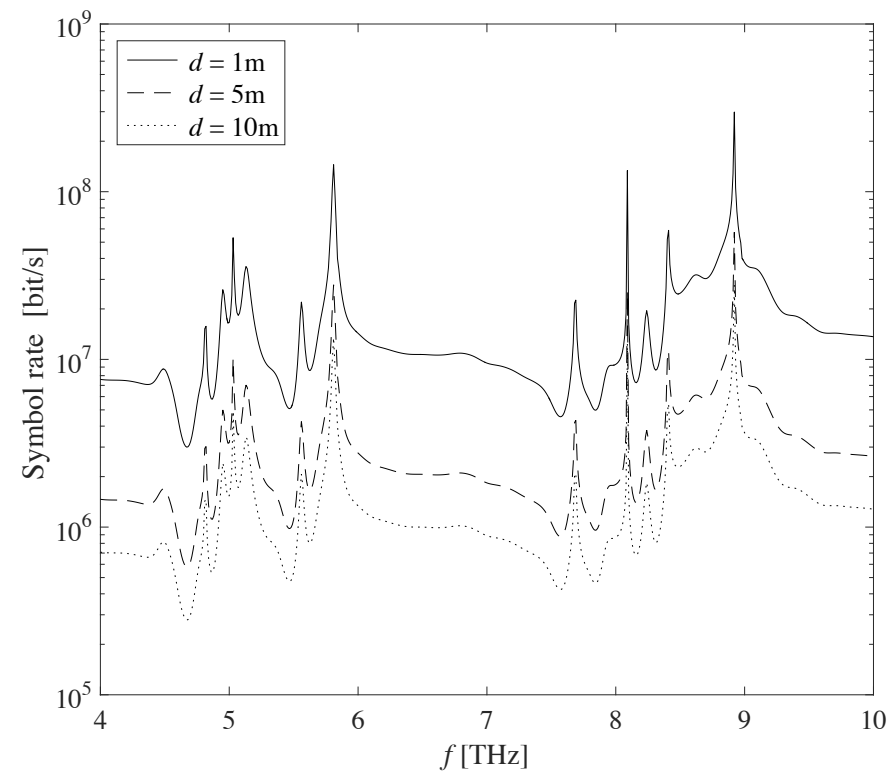

(a)

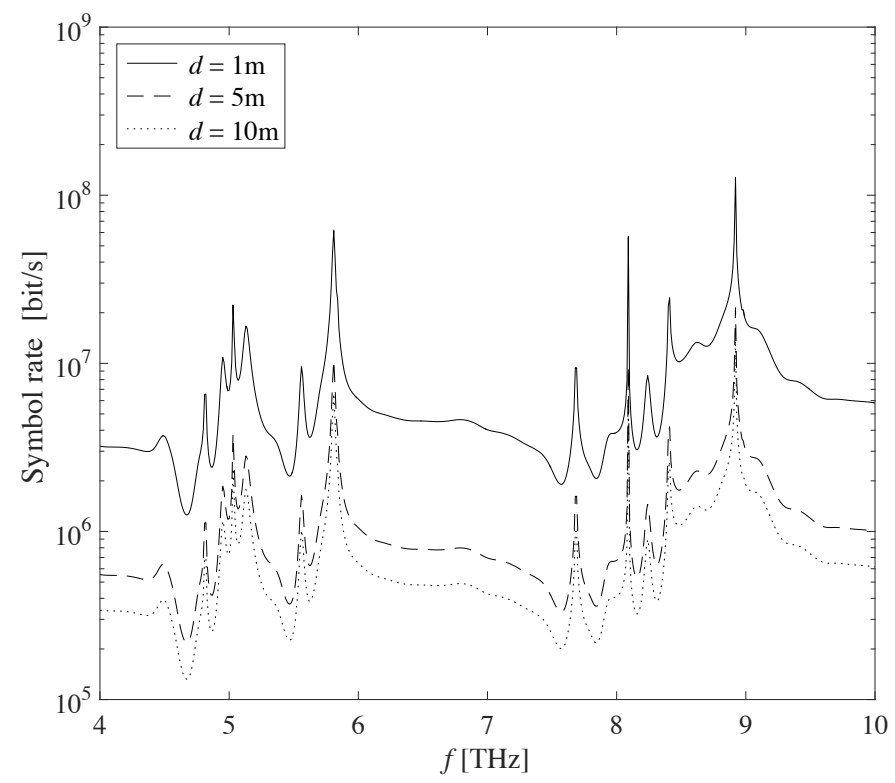

(b)

Figure 5: Symbol rates versus frequency in a chirality-affected channel with GOA, for different distances from transmitter to receiver, in case of $(a)$ one LoS and one NLoS path, an $(b)$ one LoS and five NLoS paths. 
We considered the effects of the relative chiral parameter, assuming a frequency3 dependent behavior with resonant peaks at specific frequencies. As a result, this 4 affects the channel transfer function, as well as other performances. In particular, 5 we identified the spectral windows that rise from the chiral effect, and the asso6 ciated usable bandwidths. The spectral windows vary with the distance and the 7 frequency, with corresponding bandwidths up to $6 \mathrm{THz}$, both in LoS and NLoS 8 propagation. Another contribution of the paper has been in the identification of 9 specific frequencies that allow high performance to be achieved. Thanks to the 10 frequency-dependent behavior of a chiral-metamaterial, we can tune the working 11 frequency in order to maximize the performance. Just as an example, the rms 12 delay is dependent on the distance and carrier frequency, and reaches minimum 13 values at $8.92 \mathrm{THz}$, corresponding to higher coherence bandwidths.

14 As conclusion of this paper, we can claim that GOA metamaterial present15 ing chirality effects, are really promising in terms of performance that can be 16 achieved, above all in the case of lower distances. Also, distance-adaptive and 17 multi-carrier transmissions represent the more appropriate communication tech18 niques that can benefit from the relationship between distance and bandwidth in 19 the range (4-10) THz.

\section{References}

[1] I. F. Akyildiz, J. M. Jornet, The Internet of Nano-Things, IEEE Wireless Communication Magazine 17 (6) (2010) 58-63.

[2] J. Jornet, I. Akyildiz, Channel modeling and capacity analysis for electromagnetic wireless nanonetworks in the terahertz band, Wireless 
Communications, IEEE Transactions on 10 (10) (2011) 3211-3221. doi:10.1109/TWC.2011.081011.100545.

[3] I. Llatser, A. Mestres, S. Abadal, E. Alarcon, H. Lee, A. Cabellos-Aparicio, Time- and frequency-domain analysis of molecular absorption in shortrange terahertz communications, Antennas and Wireless Propagation Letters, IEEE 14 (2015) 350-353. doi:10.1109/LAWP.2014.2362194.

[4] G. Piro, K. Yang, G. Boggia, N. Chopra, L. Grieco, A. Alomainy, Terahertz communications in human tissues at the nanoscale for healthcare applications, Nanotechnology, IEEE Transactions on 14 (3) (2015) 404-406. doi:10.1109/TNANO.2015.2415557.

[5] C. Han, A. Bicen, I. Akyildiz, Multi-ray channel modeling and wideband characterization for wireless communications in the terahertz band, Wireless Communications, IEEE Transactions on 14 (5) (2015) 2402-2412. doi:10.1109/TWC.2014.2386335.

[6] C. Zhang, C. Han, I. F. Akyildiz, Three dimensional end-to-end modeling and directivity analysis for graphene-based antennas in the terahertz band, in: 2015 IEEE Global Communications Conference (GLOBECOM), 2015, pp. 1-6. doi:10.1109/GLOCOM.2015.7417131.

[7] C. Han, A. Bicen, I. Akyildiz, Multi-wideband waveform design for distance adaptive wireless communications in the terahertz band, Signal Processing, IEEE Transactions on 64 (4) (2016) 910-922.

[8] A. Lakhtakia, R. Messier, Sculptured thin films: nanoengineerred morphology and optics, SPIE Press monograph, 2015. 
[9] F. Fang, Y. Cheng, Dual-band terahertz chiral metamaterial with giant optical activity and negative refractive index based on cross-wire structure, Progress In Electromagnetics Research M 31 (2013) 59-69.

[10] B. Wang, T. Koschny, M. Kafesaki, C. M. Soukoulis, Chiral metamaterials: Simulations and experiments, J. Opt. A: Pure Appl. Opt. 11.

[11] N. Engheta, Chiral Materials and Chiral Electrodynamics: Background \& Basic Physical Principles, in: Special Workshop on Chiral and Complex Materials Progress in Electromagnetics Research Symposium, 1991.

[12] A. Lakhtakia, Recent contributions to classical electromagnetic theory of chiral media: what next?, Speculations in Science and Technology 14 (1) (1991) 2-17.

[13] I. Lindell, A. Sihvola, S. Tretyakov, A. Viitanen, Electromagnetic waves in chiral and bi-isotropic media, Artech House, 1994.

[14] J. Zhou, D. R. Chowdhury, R. Zhao, A. Azad, H.-T. Chen, C. M. Soukoulis, A. J. Taylor, Terahertz chiral metamaterials with giant and dynamically tunable optical activity, Phys. Rev. B 86. doi:10.1103/PhysRevB.86.035448.

URL http://link.aps.org/doi/10.1103/PhysRevB.86. 035448

[15] R. Zhao, T. Koschny, C. M. Soukoulis, Chiral metamaterials: retrieval of the effective parameters with and without substrate, Optics express 18 (4).

[16] A. M. Vegni, V. Loscrí, Performance of a Chirality-affected Channel exhibiting Giant Optical Activity for Terahertz Communications, in: accepted to ACM NanoCom 2016, 2016, pp. 1-6. 